\title{
A New Methodology Based on EMD and Nonlinear Measurements for Sudden Cardiac Death Detection
}

\author{
Olivia Vargas-Lopez ${ }^{1}$, Juan P. Amezquita-Sanchez ${ }^{1,2}{ }^{\circledR}$, J. Jesus De-Santiago-Perez ${ }^{2}$, \\ Jesus R. Rivera-Guillen ${ }^{2}$, Martin Valtierra-Rodriguez ${ }^{2} \mathbb{D}$, Manuel Toledano-Ayala ${ }^{3, *}$ and \\ Carlos A. Perez-Ramirez ${ }^{1, *(D)}$ \\ 1 ENAP RG, Department of Biomedical Engineering, Faculty of Engineering, Autonomous University of \\ Queretaro, Queretaro 76144, Mexico; ovargas08@alumnos.uaq.mx (O.V.-L.); jamezquita@uaq.mx (J.P.A.-S.) \\ 2 ENAP RG, Department of Electromechanical Engineering, Faculty of Engineering, Autonomous University \\ of Queretaro, San Juan del Rio, Queretaro 76807, Mexico; sapjj@uaq.mx (J.J.D.-S.-P.); \\ jesus.rooney.rivera@uaq.mx (J.R.R.-G.); martin.valtierra@uaq.mx (M.V.-R.) \\ 3 Faculty of Engineering, Autonomous University of Queretaro, Queretaro 76100, Mexico \\ * Correspondence: toledano@uaq.mx (M.T.-A); carlos.perez@uaq.mx (C.A.P.-R.); \\ Tel.: +52-442-192-12-00 (ext. 6061)
}

Received: 13 November 2019; Accepted: 11 December 2019; Published: 18 December 2019

check for updates

\begin{abstract}
Heart diseases are among the most common death causes in the population. Particularly, sudden cardiac death (SCD) is the cause of $10 \%$ of the deaths around the world. For this reason, it is necessary to develop new methodologies that can predict this event in the earliest possible stage. This work presents a novel methodology to predict when a person can develop an SCD episode before it occurs. It is based on the adroit combination of the empirical mode decomposition, nonlinear measurements, such as the Higuchi fractal and permutation entropy, and a neural network. The obtained results show that the proposed methodology is capable of detecting an SCD episode 25 min before it appears with a 94\% accuracy. The main benefits of the proposal are: (1) an improved detection time of $25 \%$ compared with previously published works, (2) moderate computational complexity since only two features are used, and (3) it uses the raw ECG without any preprocessing stage, unlike recent previous works.
\end{abstract}

Keywords: EMD; Higuchi fractal value; entropy permutation value; SCD; ECG signal

\section{Introduction}

In the last decade, numerous methodologies or methods have been introduced in the literature for identifying several medical conditions such as diabetes [1,2], glaucoma [3,4], breast cancer [5,6], emotion detection [7], among others. Also, some proposals have been focused on the detection of several neurological disorders such as Alzheimer's disease (AD) [8-11], mild cognitive impairment (MCI) [12-15], delirium [16], epilepsy [17-19], sleep disorder [20-22], Parkinson's disease [23,24], autism spectrum disorder [25], schizophrenia [26,27], among others. These methods have been demonstrated to be efficient to diagnose a disease when it has developed symptoms in the human body. For this reason, researchers in the last years, have focused on proposing methodologies or methods capable of predicting disease or detecting it at the earliest possible state. Early identification of disease will allow enrolling the person in treatments or therapies, which can be more effective in an early stage of the disease. In this sense, various researchers have investigated the evolution conversion of MCI to AD [28], prediction of seizure [29], and prediction of sudden cardiac death (SCD) [30,31].

SCD is considered the largest cause of death in society, representing about $10 \%$ of deaths around the world [32]. An SCD episode is produced when the heart electrical conductivity changes, generating 
an inadequate blood circulation to vital organs. This condition puts at risk the patient's life if the cardiac roots are not timely attended to [33,34]. Different causes such as asystole, ventricular tachycardia, ventricular fibrillation (VF), among others, can produce an SCD episode; but, VF, represents nearly $20 \%$ of all SCD events, and is presumed the main cause of an SCD episode [35]. VF is characterized by the electrical activity disorganization in the heart ventricles, inhibiting the ability to efficiently pump blood. It can lead to death in minutes after the onset of symptoms, which is unperceivable to most people [35]. Hence, an early prediction of an SCD event in a patient or person that is suffering a VF is of vital importance because it allows receiving timely treatment and increases the survival chance.

In the last decade, researchers worldwide have presented different methods or methodologies to predict an SCD event using electrocardiogram (ECG) signals as well as the heart rate variability (HRV) [30,31,36-40]. For instance, Murukesan et al. [36] used seven nonlinear features (e.g., number of ectopic beats, among other features) combined with a support vector machine for predicting an SCD event employing HRV of the ECG signals. To validate the proposal, they employ the ECG signals from 18 and 23 patients with normal cardiac rhythm [41] and SCD [42], provided by the database named MIT-BIH, respectively. The authors report an accuracy of $96.36 \%$ two minutes before the SCD event. The following works also employ the same databases. Acharya et al., [38] combined six nonlinear features (e.g., approximate entropy, Higuchi fractal dimension, Hurst's exponent, among other features), with the discrete wavelet transform (DWT), and SVM for predicting an SCD event using the ECG signals. An accuracy of $92.11 \% 4 \mathrm{~min}$ before the onset is reported by the authors. Fujita et al., [39] explored four nonlinear features (fuzzy entropy, Renyi entropy, energy, and Tsallis entropy) and SVM for predicting an SCD event employing HRV of the ECG signals. They report that an accuracy of $94.7 \% 4$ min before the SCD event is reached. Recently, Amezquita-Sanchez et al., [31] investigated the combination of the wavelet packet transform, a nonlinear feature named homogeneity, and an enhanced probabilistic neural network for predicting an SCD episode using ECG signals. The authors report an accuracy of $95.8 \% 20 \mathrm{~min}$ before the onset, improving the window time compared with previous works in which 2, 4, and 12 min before the SCD event were studied [30,36,39]. Additionally, the authors mention that it is necessary to perform further investigation to predict an SCD event much earlier since a larger time window will allow obtaining the medical attention in a timely way to those patients that require to be transported to a hospital. Despite the promising results reported in previous work, it should be noticed that the following aspects can be improved: (1) The amount of computational resources used for the diagnosis, since computationally efficient methodologies can allow real-time predictions, (2) a larger time-window prediction, as it will allow increasing the available transportation time of a patient to the hospital, and (3) an improved efficacy. Hence, it is highly desirable to develop and apply new methodologies based on advanced signal processing techniques for predicting an SCD event with high accuracy and a good enough time window which allows a patient out of a hospital to arrive and receive timely medical procedures.

In this paper, a new methodology to predict SCD events is presented. The proposal uses as input the patient ECG signal; next, four steps are applied. In step 1, the empirical mode decomposition (EMD) method is used to separate the ECG signal into a set of frequency bands according to the information contained in the ECG signal. In step 2, Katz Index (KI) [43], Higuchi index (HI) [44], Box dimension index (BDI) [45], Shannon entropy index (SEI) [46], and permutation entropy index (PEI) [47] are employed to examine the obtained frequency bands in order to estimate features to be used for SCD prediction. In step 3, the most discriminant features are selected using an analysis of variance (ANOVA). In step 4, the selected features are classified employing a multilayer perceptron (MLP) neural network in order to predict an SCD episode $25 \mathrm{~min}$ before the onset in an automatic way. In order to validate the efficiency of the proposal, the MIT-BIH databases of the ECG data measured experimentally from 18 normal and 20 SCD patients are analyzed. 


\section{Methodology}

So as to predict the SCD onset, the methodology shown in Figure 1 is proposed. In general, it consists of the following steps. Firstly, the ECG signal is segmented in time windows of $1 \mathrm{~min}$ intervals with the aim of applying the proposed methodology for predicting if the signal corresponds to either a normal ECG signal or a signal with an SCD episode. In the next step, once the signal is segmented, the EMD method is applied to each segment in order to decompose it into a set of Intrinsic Mode Functions (IMFs), which can have information or hidden features that contribute to predicting the SCD. To quantify such features in each IMF, the KI, HI, BDI, SEI, and PEI indices are used and tested to determine which one allows distinguishing between a normal condition and an SCD condition. These indices are selected as they have proved to be sensitive to the presence of nonlinear properties in a signal [48-50]. Since the EMD method provides different IMFs and all the aforementioned indices are applied to all the IMFs, a great amount of information is generated. In order to reduce this information, the one-way ANOVA statistical method is used since this tool is useful to determine the most discriminatory indices. Finally, the selected indices are used as inputs in a Multilayer Perceptron-Neural Network (MLP-NN) to determine the SCD condition in an automatic way. A detailed description of the mathematical concepts used in the proposal and their application in the processing of ECG signals are presented in the following subsections.

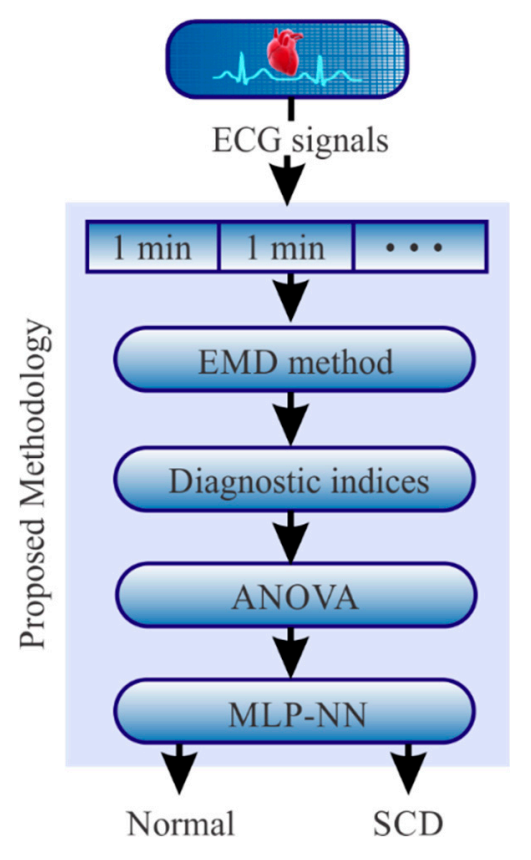

Figure 1. Proposed methodology.

\subsection{Empirical Mode Decomposition (EMD) Method}

The EMD method is a signal processing tool proposed by Huang et al., [51]. This method has been widely used in many research fields because it is capable of processing nonlinear and nonstationary signals [52]. As the features associated with the SCD condition can present nonlinear and/or nonstationary properties, the EMD method becomes a promising tool for their analysis. This method decomposes the signal under analysis in a set of mono-component signals known as IMFs. Huang et al., [51] indicate that an IMF must fulfill two conditions: i) the number of zero crossings and the number of extrema of the decomposed signal have to be either equal or different at most by one, and ii) the average value of the upper and the lower envelopes, defined by the local maxima and the local minima, respectively, is equal to zero. The algorithm to obtain the IMFs is called sifting process and consists of the following steps:

- Step 1: Obtain the extrema points (maxima and minima) of the input signal given by $\mathrm{x}(\mathrm{t})$. 
- Step 2: Construct the upper and lower envelopes with the maxima and minima, respectively, using cubic-splines.

- Step 3: Compute the mean signal from both envelopes and name it $\mathrm{m}_{1}(\mathrm{t})$.

- Step 4: Obtain the difference between $x(t)$ and $m_{1}(t)$ as $h_{1}(t)=x(t)-m_{1}(t)$.

- Step 5: Check if $h_{1}(t)$ is an IMF (conditions $i$ and ii). If $h_{1}(t)$ is not an IMF, steps from 1 to 4 are repeated in a new k-iteration; otherwise, $h_{k}(t)$ becomes the first IMF, i.e., $\mathrm{IMF}_{1}$.

- $\quad$ Step 6: Obtain the residual signal between $x(t)$ and $\mathrm{IMF}_{1}$ as $\mathrm{r}_{1}(\mathrm{t})=\mathrm{x}(\mathrm{t})-\mathrm{IMF}_{1}$.

- Step 7: Check if $r_{1}(t)$ is a monotonic function, i.e., a signal from which no more IMFs can be extracted. If $r_{1}(t)$ is not a monotonic signal, $r_{1}(t)$ becomes $x(t)$ and the aforementioned steps are repeated in order to obtain the remaining IMFs. The process ends when $r_{k}(t)$ becomes a monotonic function.

At the end, the input signal, $x(t)$, is decomposed into $N$-IMFs and a final residue, $r_{n}(t)$, as follows:

$$
x(t)=\sum_{\mathrm{n}=1}^{N} I M F_{\mathrm{n}}+r_{\mathrm{N}}(t)
$$

In the proposal, each $1 \mathrm{~min}$ interval of the ECG signal, $x(t)$, is decomposed in its IMFs, which are analyzed by the proposed diagnostic indices.

\subsection{Diagnostic Indices}

The early and accurate prediction of an SCD requires extracting the nonlinear features of the ECG signal. The EMD splits the ECG information into frequency bands (IMFs) whose need being quantified in order to predict an SCD episode. In this work, several diagnostic indexes such as KI, HI, BDI, SEI, and PEI are tested to select the ones that better suit the requirements of the SCD prediction.

\subsubsection{Entropy}

In general, entropy attempts to quantify the randomness of a signal [47]. In the proposed methodology, the entropy index could be a useful tool to detect the random behavior in the ECG signal and predict the SCD. Two approximations to the entropy are analyzed.

\section{Shannon Entropy Index (SEI)}

SEI is a logarithmic value used to indicate how much the measured process changes [46]. In particular, the range of values that SI can take depends on the number of bits used in analog-to-digital converter (ADC). For instance, the SEI for 12 bits ADC ranges from 0 to 12 where a low value, such as DC voltage, means the signal takes few values along time. Conversely, a value near 12 indicates that the signal takes all the possible values of the ADC. In other words, high SEI indicates that all the possible values of the ADC can occur with the same probability. Considering $M$ possible values, $v_{M}$, for each $I M F_{n}=\left\{v_{1}, v_{2}, v_{3}, \ldots, v_{M}\right\}$ the SEI is calculated by means of Equation (2).

$$
S E I_{n}=-\sum_{i=1}^{M}\left[f\left(v_{i}\right) / M\right] \log _{2}\left(f\left(v_{i}\right) / M\right)
$$

where $f\left(v_{i}\right)$ is the frequency value $v_{i}$ of the $I M F_{n}$.

Permutation Entropy Index (PEI)

As the ECG signal can be considered as a nonlinear time series, the PEI gives a complexity measurement for this type of signal with a high-noise immunity [12]. The normalized PEI quantifies the regularity of the analyzed signal in a range from 0 to 1 , where 0 indicates high regularity in the 
series and 1 indicates the opposite regularity. Defining $\pi$ as an order pattern of permutations that can occur in the analyzed $I M F_{n}$, the PEI is determined by Equation (3).

$$
P I_{n}=-\frac{1}{\ln (m !)} \sum_{i=1}^{m !} p(\pi) \ln (p(\pi))
$$

where $\mathrm{m}$ is the size of $\pi$ and $p(\pi)$ is the probability that the pattern $\pi$ occurs in the $I M F_{n} ; p(\pi)$ is calculated using Equation (4).

$$
p(\pi)=f(\pi) /[K-(m-1) \tau]
$$

In this case, $f(\pi)$ is the frequency of the pattern $\pi$ in the $I M F_{n}$ and $\tau$ is the time lag.

\subsection{Fractal Dimension (FD)}

A self-similar signal is considered exactly or approximately similar to a part of itself. FD provides information about the geometrical structure at multiples scales of a self-similar signal [52]. A small FD indicates low complexity in the signal; conversely, a high FD indicates a high complexity in the signal. Then, if the ECG signal is self-similar, FD can provide information about its geometry. Thus, if geometrical changes occur when a person suffers an SCD event, they will be reflected in the FD value, making it a potential index for SCD prediction. Diverse FD methods have been reported in the literature, which can measure the geometrical self-similarities of a signal in a different manner. Hence, in this work three approaches, KI, HI, and BDI, are evaluated to determine if they are capable of identifying patterns to be associated with the rhythm cardiac using ECG signals.

\subsubsection{Katz Index (KI)}

The KI of a time signal is defined as Equation (5) [43]:

$$
K I_{n}=\frac{\log _{10}\left(L_{n} / a\right)}{\log _{10}(d / a)}
$$

where $L_{n}$ is the total length of the analyzed IMF. This parameter is calculated by means of Equation (6).

$$
L_{n}=\sum_{k=2}^{K} \operatorname{distance}\left(I M F_{n}(k)-I M F_{n}(k-1)\right)
$$

In Equation (5), $a$ is the average of the total length of the analyzed IMF and is calculated using Equation (7).

$$
a=\frac{L_{n}}{K-1}
$$

Finally, $d$ is the maximum distance between the first value of the IMF and all the possible values of the IMF.

\subsubsection{Higuchi Index (HI)}

The Higuchi's FD approach requires being computed in several steps [44]:

- $\quad$ Step 1. Decompose the analyzed IMF into several discrete-time sequences $S_{p}^{q}$

$$
S_{p}^{q}=\left\{\operatorname{IMF}(q), \operatorname{IMF}(q+p), \operatorname{IMF}(q+2 p), \cdots, \operatorname{IMF}\left(q+\left\lfloor\frac{K-q}{p}\right\rfloor p\right)\right\}
$$

where $q=1,2,3, \ldots, Q_{\max }, q$ and $p$ are integer values which determine the initial IMF value and the number of skipped samples, respectively. $Q_{\max }$ is the maximum number of skipped samples. 
- $\quad$ Step 2. Calculate the length of the generated sequences $S_{p}^{q}$ using Equation (9).

$$
L_{q}(p)=\left\{\left(\sum_{i=1}^{\left\lfloor\frac{K-q}{p}\right\rfloor}\left|S_{p}^{i}-S_{p}^{i-1}\right|\right) \frac{K-1}{\left\lfloor\frac{K-q}{p}\right\rfloor p}\right\}
$$

- $\quad$ Step 3. Compute the sum of all the generated sequences by means of Equation (10).

$$
L(p)=\sum_{q=1}^{p} L_{q}(p)
$$

- Step 4. Calculate the slope of the line that best fits the plotted plane $(\ln (1 / q), \ln (\mathrm{L}(q))$. The obtained slope is the HI value.

\subsubsection{Box Dimension Index (BDI)}

The BDI of a time-series signal, in this case, an IMF, is calculated using Equation (11) [45]

$$
B D I_{n}=-\frac{\ln \left(\sum_{k=1}^{K-1}\left|I M F_{n}(k+1)-I M F_{n}(k)\right| / \Delta t\right)}{\ln (\Delta t)}
$$

where $\Delta t$ is the sampling-time interval and $K$ is the number of samples of the IMF.

\subsection{The Analysis of Variance (ANOVA)}

ANOVA is a statistical tool to analyze sets of quantitative data obtained from various experimental conditions or treatments. One-way ANOVA performs the comparison of two or more statistical means of several populations. The comparison is made by means of a test of hypotheses where a null hypothesis $H o$ is established, the null hypothesis claims that there is no significant difference between the sample means of the analyzed populations [38]. ANOVA generates a test statistic $f$, which determines if the null hypothesis is accepted or rejected at a specific level of significance according to Fisher's distribution. In statistical packages, the ANOVA generates a table with the analysis of the data, the test statistic, and a probability $p$-value. This $p$-value represents the minimum value of the significance for which the null hypothesis can be rejected. Small $p$-values allow us to easily reject the null hypothesis, indicating that there is a significant difference between the population means of the analyzed data sets.

The ability of ANOVA to differentiate different groups is used in this work to select the entropy and FD indicators that are statistically different for normal individuals and those who presented an SCD. The comparison of the proposed indicators between both groups is done with the objective of reducing the number of indicators, i.e., those that can better characterize both study groups.

\subsection{Multilayer Perceptron (MLP)}

Finally, the most discriminant nonlinear indices that are selected by the ANOVA method are used to train an artificial neural network in order to predict an SCD event in an automatic manner. Neural networks are powerful mathematical tools that simulate models of learning and decision, similar to the behavior of biological brains. The Artificial Neural Networks (ANNs) collect information of various input and output variables, from which the learning and interaction models are built. The input information is processed in different layers of the neural network in order to generate the output values called activation variables. In each layer, there are neurons interconnected by links, in these links there are output values for each neuron, which are multiplied by one weight. The objective of the neural network is to identify patterns and to make decisions on problems that are usually difficult to solve 
in an analytical way. This tool is widely used in problems of machine learning, pattern recognition and artificial vision, among others [31,53,54]. A multilayer perceptron is a type of feedforward neural network that consists of three main layers: an input layer, one or more hidden layers, and an output layer [52]. This configuration of the MLP allows solving problems that are not linearly separable, capturing the nonlinear relationship in complex data [55].

The output in each neuron is obtained by adding a weighted set of inputs. This sum is defined by Equation (12).

$$
S_{j}=\sum_{i=1}^{n} w_{i j} x_{i}+b_{j}
$$

where $w_{i j}$ represents the weighting factors corresponding to the input $x_{i}$ in the layer $j$ and $b_{j}$ is the bias unit added to each neuron. The output $y_{j}$ is determined by a transfer function $F$, which is defined as:

$$
y_{j}=F\left(S_{j}\right)=\frac{1}{1+e^{-S_{j}}} .
$$

This transfer function is the sigmoid bipolar. It is employed in this work since it can deal with nonlinear features [52], which can be found in the ECG signals.

\section{Materials}

In order to validate and test the proposal, ECG signals of two international databases are used. The first one is the Normal Sinus Rhythm (NSR) database, MIT/BIH-NSR [41], which includes the ECG signals of 18 subjects. Experts from the Arrhythmia Laboratory at Boston's Beth Israel Hospital confirmed that the subjects presented a normal heart rate; hence, these signals are taken as ECG signals without properties associated with the SCD condition, i.e., normal condition. The second one is the SCDH database, MIT/BIH-SCDH [42], which includes the ECG signals of 23 subjects with SCD produced by ventricular fibrillation. For these signals, the exact time of the SCD is indicated. This time is used as a reference to predict the appearance of SCD in an earlier way. It is important to mention that only the ECG signals of 18 subjects are used in the analysis because the remaining subjects present other heart alterations such as hypertrophic cardiomyopathy and ventricular tachycardia (future opportunities of research). Table 1 summarizes some features of the used ECG signals; a more detailed description can be found in Amezquita-Sanchez et al., [31].

Table 1. Information about sudden cardiac death (SCD) and normal databases.

\begin{tabular}{cccc}
\hline Database & Group & Subjects (Sex) & Age \\
\hline NSR & Normal & $18(13$ female $)$ & $35 \pm 15$ \\
SCDH & SCD & 23 (8 female) & $49.5 \pm 32.5$ \\
\hline
\end{tabular}

As the ECG signals correspond to different databases, some aspects have to be considered. For instance, in the SCDH database (SCD group), a sampling frequency of $250 \mathrm{~Hz}$ was used, whereas a sampling frequency of $128 \mathrm{~Hz}$ was used in the NSR database (normal group). For consistency, the ECG signals with $250 \mathrm{~Hz}$ are down-sampled to $128 \mathrm{~Hz}$. Figure 2 shows the ECG signals for both a person with a normal condition and a person with SCD condition. In these signals, the goal is to detect features that allow predicting the SCD using time windows of $1 \mathrm{~min}$, as used by other authors [36-40]. This window time size is selected to continuously analyze so the patient condition status can be frequently monitored; in this way, medical help can be obtained. Although the ECG signals were monitored for $24 \mathrm{~h}$, only $25 \mathrm{~min}$ of them before the SCD occurrences are analyzed. The efforts of this research are put in this time window since it outperforms the reported times by other researchers around the world, providing an earlier SCD prediction. For the ECG signals of the normal group, a 1 min interval of each signal is randomly selected. 
(a)

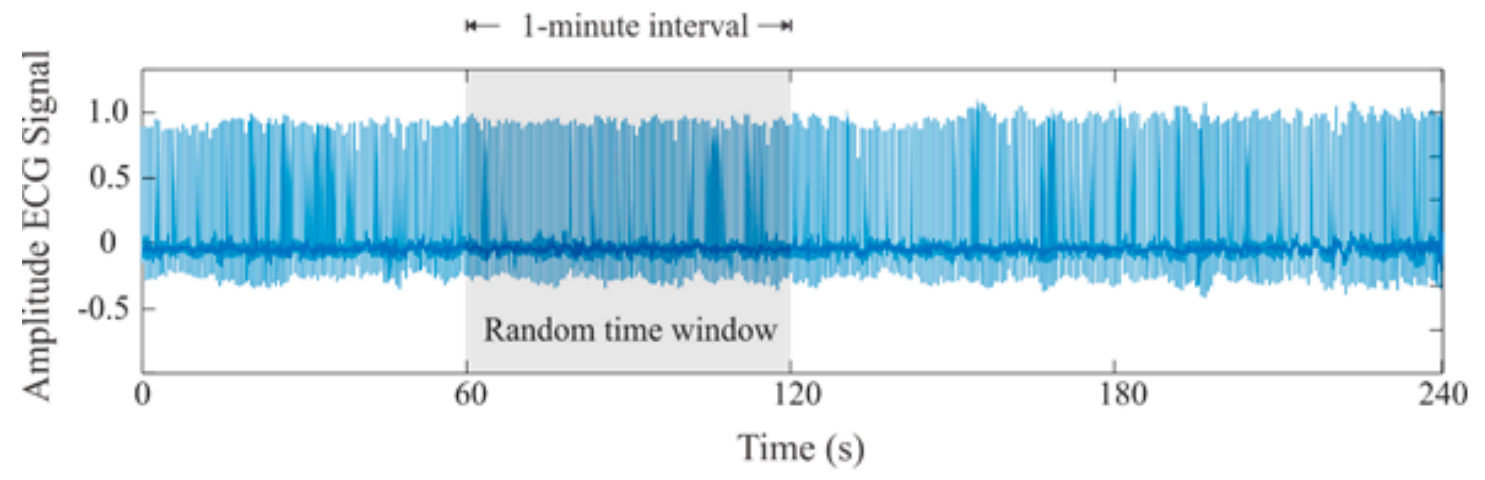

(b)

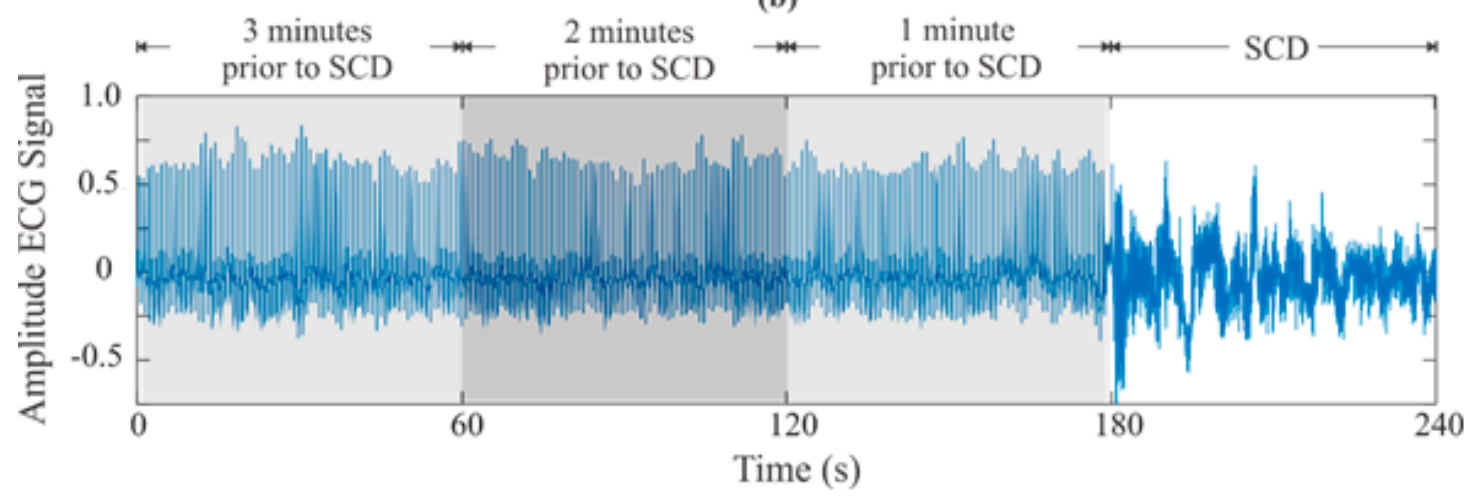

Figure 2. ECG signals for a: (a) healthy subject and (b) subject with an SCD episode.

\section{Experimentation and Results}

Following the steps of the proposed methodology, the 1 min intervals of both the healthy and the SCD group signals are decomposed using the EMD method, where the first six IMFs are used. It should be noticed that the last IMFs are generated due to interpolation errors; thus, they do not contain physical useful information and can be safely discarded for further analysis [56]. To illustrate the analyzed signals, Figure 3 shows the six obtained IMFs for a normal subject and an SCD subject one minute prior to its development. From the figure, it is seen that no visual differences associated with the SCD condition can be detected. In other words, the perceived differences are not produced by the SCD condition [40]; instead, they can be related to the gender, age, or the patient physical condition, among other causes.

After obtaining the IMFs, the five diagnostic indices (KI, HI, BDI, SEI, PEI) are obtained for each IMF. This results in the estimation of 30 indices for each patient and condition. In other words, 480 values are calculated for each 1 min interval. All the obtained values are analyzed using the one-way ANOVA method in order to determine which ones are the most discriminant features. The obtained results show that the PEI value from IMF 5 and HI value from IMF 3 are the ones that have the highest statistical significance, since a low $p$-value is obtained for each index. Consequently, it can be affirmed that both values have the necessary information to distinguish from a person that has a normal heart signal from one that suffers from an SCD episode without considering their age or general physical condition.

Figure $4 a$,b show the HI value distribution using IMF 3 and the PEI value distribution using IMF 5, respectively. The normal patients are denoted by the letter $\mathrm{N}$, whereas the remaining boxes show the PEI and HI values, respectively, in $1 \mathrm{~min}$ intervals up to $25 \mathrm{~min}$ before the SCD event. From these figures, it can be seen that there is an evident difference between the values from the $\mathrm{N}$ group and the group with an increased risk of suffering an SCD episode. In particular, there are some values that are far from the central zone (denoted by the + symbol), which most of the time fall outside of the zone for 
normal patients. It should be noticed that the ECG signals can be affected by several factors such as age, body positions, the person's emotional health, among others [31]. These factors yield to have different cardiac rhythms; thus, different measured waveforms are produced and measured. Nevertheless, the combination of the two indices allows obtaining enough information to generate an accurate classifier.

Table 2 presents a summary of the mean, standard deviation, and $p$-value for the two indices, from $1 \mathrm{~min}$ to $25 \mathrm{~min}$ before the SCD episode in $1 \mathrm{~min}$ intervals. It can be seen that the $p$-value is lower than 0.00001 for all the intervals. After the values are selected (PEI from IMF 5 and HI from IMF 3), an ANN-based classifier is designed. To this purpose, $45 \%$ of the available data, i.e., eight patients for each class, were randomly selected and the maximum and minimum values for each 1 min interval of these data were obtained to generate 400 random values within the aforementioned limits. This procedure allows ensuring the necessary diversity to capture all the possible values that can indicate the existence of an SCD event. To obtain the weights and biases, the Levenberg-Marquardt algorithm was employed. Lastly, the classifier was validated using the last 55\% of the data, where the patients were also randomly selected. Table 3 shows the accuracy obtained for each interval, where it can be seen that the proposal achieves good accuracy. An average of $94 \%$ up to 25 min before the occurrence of an SCD event was obtained with the proposed methodology. This time window allows the patient to take the proper remedial actions (medical treatment), increasing the survival chance.

(a)
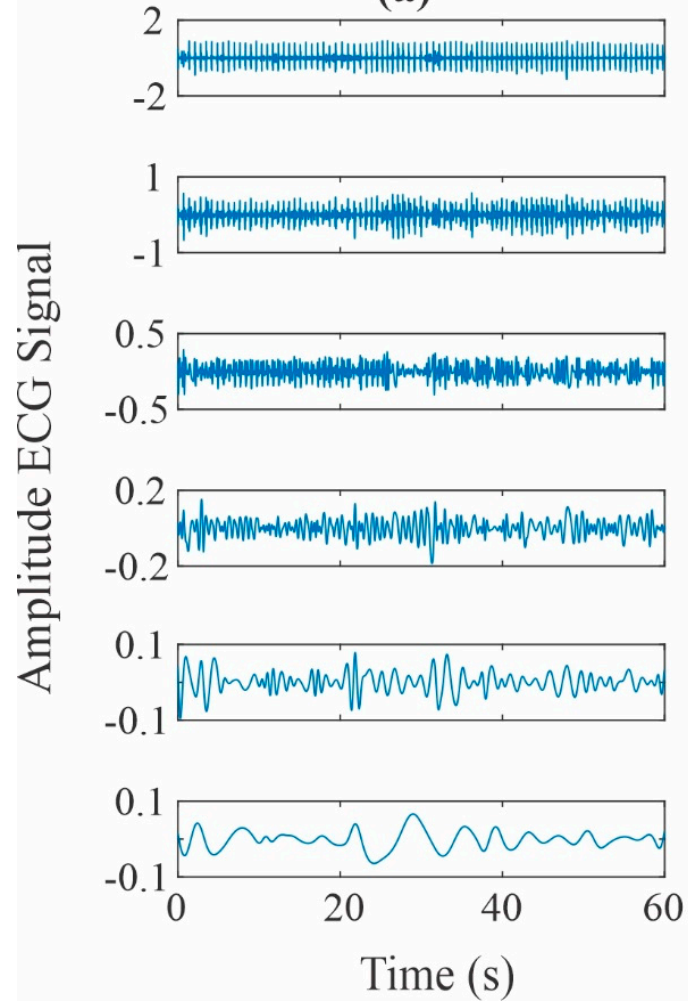

(b)
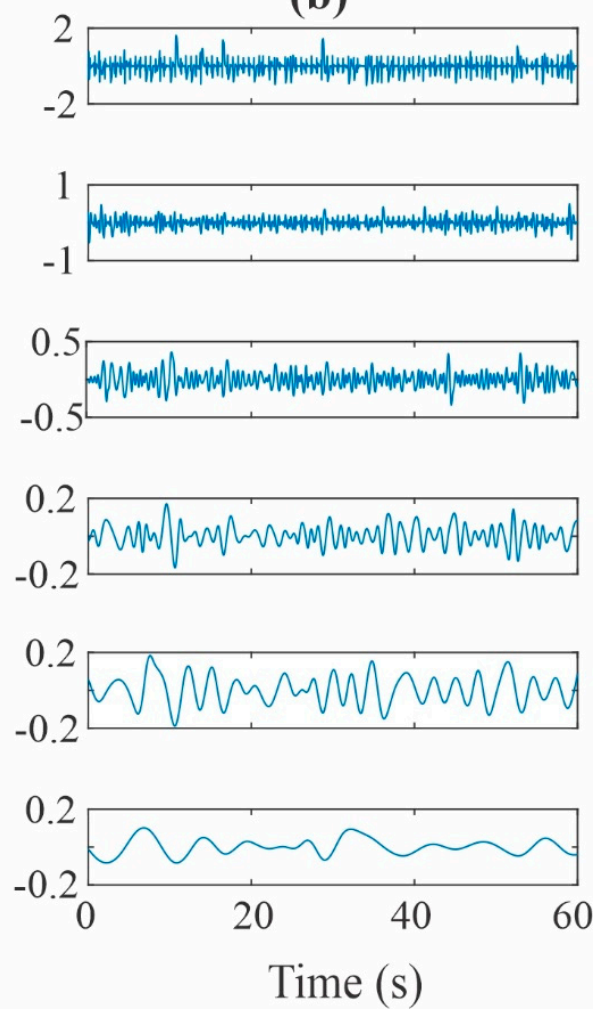

Figure 3. IMF decompositions obtained from an ECG signal of a: (a) healthy subject and (b) subject with an SCD episode. 
(a)

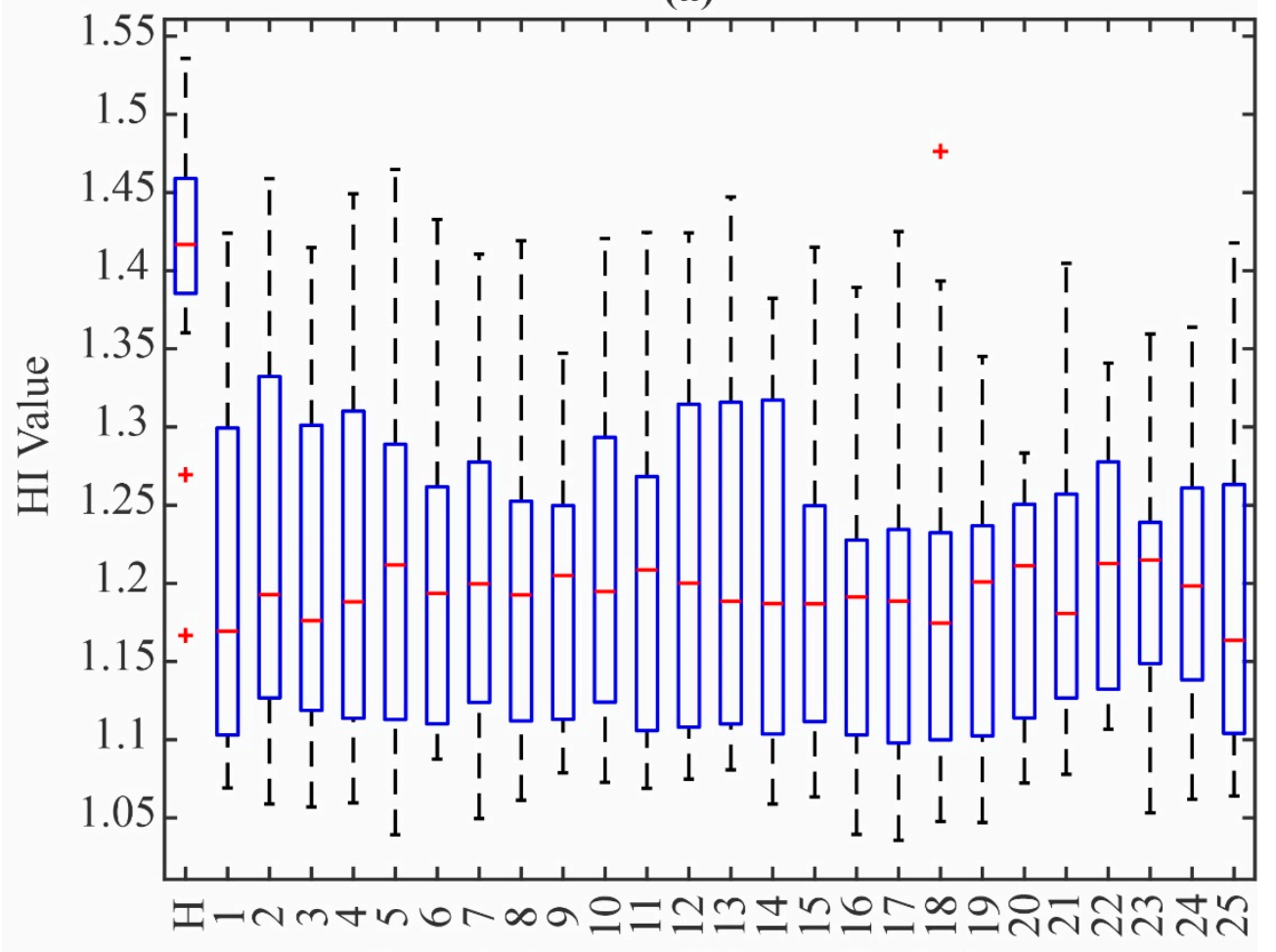

Time before an SCD eposide (min)

(b)

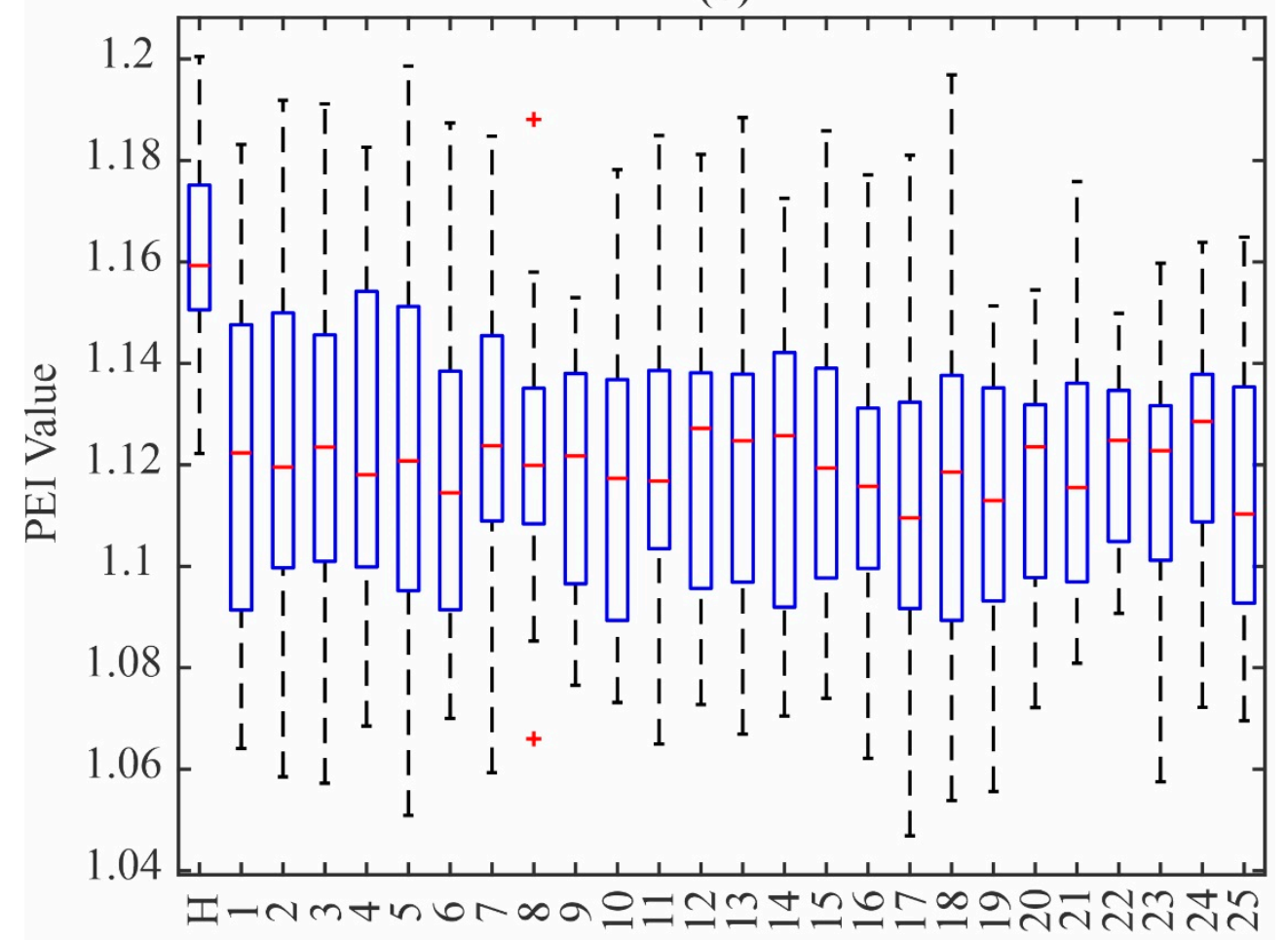

Time before an SCD eposide (min)

Figure 4. Box plots for (a) Higuchi index (HI) from IMF 3 and (b) permutation entropy index (PEI) from IMF 5. 
Table 2. Information about the SCD and normal databases.

\begin{tabular}{|c|c|c|c|c|c|}
\hline \multicolumn{2}{|c|}{ Normal } & \multirow[b]{2}{*}{ Minute } & \multicolumn{3}{|c|}{ SCD } \\
\hline Fractal & $\mu \pm \sigma$ & & Fractal & $\mu \pm \sigma$ & $p$-Value \\
\hline \multirow{50}{*}{$\begin{array}{l}\text { HI } \\
\text { PEI }\end{array}$} & $1.4061 \pm 0.0892$ & \multirow{2}{*}{25} & $\mathrm{HI}$ & $1.1875 \pm 0.1077$ & $4.71 \times 10^{-07}$ \\
\hline & $1.0793 \pm 0.0143$ & & PEI & $1.0505 \pm 0.0126$ & $1.25 \times 10^{-06}$ \\
\hline & & \multirow{2}{*}{24} & $\mathrm{HI}$ & $1.2055 \pm 0.1231$ & $3.77 \times 10^{-07}$ \\
\hline & & & PEI & $1.0556 \pm 0.0138$ & $4.42 \times 10^{-05}$ \\
\hline & & \multirow{2}{*}{23} & $\mathrm{HI}$ & $1.2014 \pm 0.1187$ & $1.71 \times 10^{-07}$ \\
\hline & & & PEI & $1.0533 \pm 0.0128$ & $7.37 \times 10^{-06}$ \\
\hline & & \multirow{2}{*}{22} & $\mathrm{HI}$ & $1.2050 \pm 0.1082$ & $2.16 \times 10^{-07}$ \\
\hline & & & PEI & $1.0528 \pm 0.0113$ & $2.28 \times 10^{-06}$ \\
\hline & & \multirow{2}{*}{21} & $\mathrm{HI}$ & $1.1948 \pm 0.1051$ & $1.02 \times 10^{-06}$ \\
\hline & & & PEI & $1.0517 \pm 0.0110$ & $1.02 \times 10^{-06}$ \\
\hline & & \multirow{2}{*}{20} & $\mathrm{HI}$ & $1.1885 \pm 0.1038$ & $2.31 \times 10^{-08}$ \\
\hline & & & PEI & $1.0539 \pm 0.0119$ & $6.28 \times 10^{-06}$ \\
\hline & & \multirow{2}{*}{19} & $\mathrm{HI}$ & $1.1829 \pm 0.0874$ & $8.24 \times 10^{-08}$ \\
\hline & & & PEI & $1.0498 \pm 0.0114$ & $4.11 \times 10^{-07}$ \\
\hline & & \multirow{2}{*}{18} & $\mathrm{HI}$ & $1.1943 \pm 0.1167$ & $5.04 \times 10^{-06}$ \\
\hline & & & PEI & $1.0507 \pm 0.0191$ & $4.19 \times 10^{-05}$ \\
\hline & & \multirow{2}{*}{17} & $\mathrm{HI}$ & $1.1915 \pm 0.1065$ & $1.50 \times 10^{-06}$ \\
\hline & & & PEI & $1.0507 \pm 0.0163$ & $1.09 \times 10^{-05}$ \\
\hline & & \multirow{2}{*}{16} & $\mathrm{HI}$ & $1.1902 \pm 0.1173$ & $8.04 \times 10^{-07}$ \\
\hline & & & PEI & $1.0521 \pm 0.0166$ & $2.51 \times 10^{-05}$ \\
\hline & & \multirow{2}{*}{15} & $\mathrm{HI}$ & $1.1925 \pm 0.1270$ & $6.35 \times 10^{-07}$ \\
\hline & & & PEI & $1.0527 \pm 0.0136$ & $7.55 \times 10^{-06}$ \\
\hline & & \multirow{2}{*}{14} & $\mathrm{HI}$ & $1.2034 \pm 0.1191$ & $6.55 \times 10^{-06}$ \\
\hline & & & PEI & $1.0511 \pm 0.0151$ & $7.00 \times 10^{-06}$ \\
\hline & & \multirow{2}{*}{13} & $\mathrm{HI}$ & $1.2160 \pm 0.1026$ & $3.07 \times 10^{-05}$ \\
\hline & & & PEI & $1.0550 \pm 0.0147$ & $4.98 \times 10^{-05}$ \\
\hline & & \multirow{2}{*}{12} & $\mathrm{HI}$ & $1.2165 \pm 0.1071$ & $1.54 \times 10^{-05}$ \\
\hline & & & PEI & $1.0544 \pm 0.0146$ & $3.42 \times 10^{-05}$ \\
\hline & & \multirow{2}{*}{11} & $\mathrm{HI}$ & $1.2086 \pm 0.1126$ & $3.37 \times 10^{-06}$ \\
\hline & & & PEI & $1.0516 \pm 0.0165$ & $1.92 \times 10^{-05}$ \\
\hline & & \multirow{2}{*}{10} & $\mathrm{HI}$ & $1.2160 \pm 0.1241$ & $1.42 \times 10^{-05}$ \\
\hline & & & PEI & $1.0531 \pm 0.0172$ & $5.66 \times 10^{-05}$ \\
\hline & & & $\mathrm{HI}$ & $1.1960 \pm 0.0905$ & $1.85 \times 10^{-07}$ \\
\hline & & 9 & PEI & $1.0527 \pm 0.0125$ & $4.16 \times 10^{-06}$ \\
\hline & & & $\mathrm{HI}$ & $1.2052 \pm 0.0742$ & $2.01 \times 10^{-06}$ \\
\hline & & 8 & PEI & $1.0549 \pm 0.0180$ & $8.71 \times 10^{-05}$ \\
\hline & & & $\mathrm{HI}$ & $1.2114 \pm 0.0923$ & $3.75 \times 10^{-06}$ \\
\hline & & 7 & PEI & $1.0557 \pm 0.0164$ & $8.92 \times 10^{-05}$ \\
\hline & & & $\mathrm{HI}$ & $1.2039 \pm 0.0811$ & $2.66 \times 10^{-06}$ \\
\hline & & 6 & PEI & $1.0524 \pm 0.0156$ & $1.88 \times 10^{-05}$ \\
\hline & & & $\mathrm{HI}$ & $1.2076 \pm 0.0819$ & $8.69 \times 10^{-06}$ \\
\hline & & 5 & PEI & $1.0541 \pm 0.0184$ & $1.55 \times 10^{-04}$ \\
\hline & & 4 & $\mathrm{HI}$ & $1.2144 \pm 0.0861$ & $2.07 \times 10^{-05}$ \\
\hline & & 4 & PEI & $1.0547 \pm 0.0172$ & $1.21 \times 10^{-04}$ \\
\hline & & & $\mathrm{HI}$ & $1.2052 \pm 0.1038$ & $2.85 \times 10^{-06}$ \\
\hline & & 3 & PEI & $1.0546 \pm 0.0184$ & $9.37 \times 10^{-05}$ \\
\hline & & & $\mathrm{HI}$ & $1.2192 \pm 0.1194$ & $2.23 \times 10^{-05}$ \\
\hline & & 2 & PEI & $1.0553 \pm 0.0197$ & $9.24 \times 10^{-04}$ \\
\hline & & & $\mathrm{HI}$ & $1.2019 \pm 0.1108$ & $2.87 \times 10^{-06}$ \\
\hline & & 1 & PEI & $1.0530 \pm 0.0168$ & $4.29 \times 10^{-05}$ \\
\hline
\end{tabular}


Table 3. Obtained accuracy for each 1 min interval.

\begin{tabular}{cc}
\hline Minute & Accuracy \\
\hline 1 & $90 \%$ \\
2 & $100 \%$ \\
3 & $90 \%$ \\
4 & $100 \%$ \\
5 & $90 \%$ \\
6 & $100 \%$ \\
7 & $90 \%$ \\
8 & $100 \%$ \\
9 & $100 \%$ \\
10 & $90 \%$ \\
11 & $90 \%$ \\
12 & $90 \%$ \\
13 & $100 \%$ \\
14 & $90 \%$ \\
15 & $100 \%$ \\
16 & $90 \%$ \\
17 & $90 \%$ \\
18 & $100 \%$ \\
19 & $90 \%$ \\
20 & $100 \%$ \\
21 & $100 \%$ \\
22 & $90 \%$ \\
23 & $90 \%$ \\
24 & $100 \%$ \\
25 & $100 \%$ \\
\hline & \\
&
\end{tabular}

\section{Results Discussion}

The obtained results show that an average accuracy of $94 \%$ is obtained to predict 25 min before an SCD event occurs, which is a relatively long period of time [57-59]. In general terms, it is desirable to have a classifier that can have a $100 \%$ accuracy, as this will indicate that both all healthy patients and the ones with an SCD episode are correctly identified; yet, if this situation is not possible, a classifier that has a low number of false-negative detections, i.e., patients that are identified as healthy but they have an SCD episode, is acceptable. On the contrary, if the classifier has a high number of false-negative cases, patients that require immediate medical help will not be identified, increasing the risks of having a life-threatening event. As Amezquita-Sanchez et al., [31] note, the prediction of the SCD event is a challenge as the ECG signal has subtle changes that the diagnostic indices must detect; moreover, it is well known that ECG signals have a nonlinear behavior $[31,33,36,39,40]$. In this sense, the indices used in this work can properly deal with nonlinear signals [12], which coupled with the ANN classifier allows generating a methodology that can indicate the possibility of having an SCD event in a timely way. Nevertheless, it is important to continue improving the classifier accuracy in order to achieve a $100 \%$ accuracy, as this will indicate that neither false-positives (patients that are identified with an SCD episode but are healthy) nor false-negatives are detected, making the proposal a reliable and efficient tool to help physicians in the diagnosis process.

Table 4 presents a comparison between recent articles that uses the same database. The table includes the minutes before the SCD event occurs, the accuracy obtained, and a brief description of the proposed methodology. It can be seen that the proposed methodologies that obtain the highest accuracy values make use of algorithms such as different types of entropies, Hurst exponent, correlation dimension, among others, that can process nonlinear signals; nevertheless, they require the utilization of many indices to perform the prediction, which in consequence increases the required computational time to deliver the result. In contrast, the proposed methodology only uses two indices, which leads to a decrease in the used computational time. On the other hand, it should be noticed that the proposal 
can predict the likelihood of the SCD event up to $25 \mathrm{~min}$ before its appearance, which improves the detection time presented by Amezquita-Sanchez et al., [31] by a margin of $5 \mathrm{~min}$. The development of methods that can expand the detection time of the SCD episodes before its occurrence has been one of the goals of the researchers in this field. Since the detection of the ECG waveform changes before the SCD event increases its difficulty when the time before the SCD episode occur increases; thus, the development of signal processing techniques that can detect those changes also becomes a challenging task. In this sense, the proposal increases $25 \%$ the detection time using only two indices, which is a reasonable compromise to allow performing an on-line operation. It should be noticed that the proposal obtains the features used for classification from the raw ECG signal, unlike other methodologies that make use of the HRV signal, which increases the computational time required to execute the proposed methodologies, as they have to use further signal processing strategies to correctly detect the heartbeats required to estimate the aforementioned signal [60].

Table 4. Comparison with similar works.

\begin{tabular}{|c|c|c|c|}
\hline Author & Signal & Methodology & $\begin{array}{l}\text { Prediction Time } \\
\text { (Accuracy) }\end{array}$ \\
\hline Shen et al., (2007) [61] & ECG & $\begin{array}{l}\text { - Three features used as factors to predict the SCD } \\
\text { are: (1) HRV mean, (2) the ratio Low } \\
\text { frequency/High frequency, and (3) Very } \\
\text { low frequency. } \\
\text { - An artificial neural network is used as classifier. }\end{array}$ & $\begin{array}{c}2 \min \\
(67.44 \%)\end{array}$ \\
\hline $\begin{array}{l}\text { Ebrahimzadeh and } \\
\text { Pooyan (2011) [62] }\end{array}$ & HRV & $\begin{array}{l}\text { - Three analyses are carried out. The first one uses } \\
\text { features from both the time and } \\
\text { frequency-domain. The second one is a TF } \\
\text { domain analysis, in which } 10 \text { features are } \\
\text { extracted (maximum energy, minimum energy, } \\
\text { the difference between maximum and minimum } \\
\text { in each window, standard deviation between } \\
\text { energy of time windows, the total and average } \\
\text { energy of signal in the very low frequency (VLF), } \\
\text { low frequency (LF), and high frequency (HF) } \\
\text { bands. The last analysis, a nonlinear one, uses } \\
\text { two different parameters from the RR intervals: } \\
\text { (1) Poincaré and (2) Detrended fluctuation } \\
\text { analysis (DFA). } \\
\text { The K-nearest neighbor is used as classifier. }\end{array}$ & $\begin{array}{c}4 \min \\
(83.96 \%)\end{array}$ \\
\hline $\begin{array}{c}\text { Ebrahimzadeh et al., } \\
\text { (2014) }\end{array}$ & HRV & $\begin{array}{l}\text { - } 5 \text { features in time-domain are extracted: mean of } \\
\text { all NN intervals (MNN), standard deviation of all } \\
\text { NN intervals (SDNN), the square root of the } \\
\text { mean of the sum of the squares of differences } \\
\text { between adjacent NN Intervals (RMSSD), the } \\
\text { standard deviation of differences between } \\
\text { adjacent NN intervals (SDSD), the proportion } \\
\text { derived by dividing the number of interval } \\
\text { differences of NN intervals greater than } 50 \mathrm{~ms} \text { by } \\
\text { the total number of NN intervals (PNN50). } 4 \\
\text { features in frequency-domain (VLF, LF, HF and } \\
\text { the ratio LF/HF) are also employed. } \\
\text { Principal component analysis is used to select the } \\
\text { most relevant features. } \\
\text { An MLP is used as classifier. }\end{array}$ & $\begin{array}{c}1 \mathrm{~min} \\
(99.16 \%)\end{array}$ \\
\hline
\end{tabular}


Table 4. Cont.

\begin{tabular}{|c|c|c|c|}
\hline Author & Signal & Methodology & $\begin{array}{l}\text { Prediction Time } \\
\text { (Accuracy) }\end{array}$ \\
\hline Acharya et al., (2015) & ECG & $\begin{array}{l}18 \text { nonlinear features are extracted by using } \\
\text { different methods such as Dimension fractal (FD), } \\
\text { Hurst exponent (H), approximate entropy } \\
\text { (ApproxEnt), Sample entropy (SampEnt), } \\
\text { Detrended fluctuation analysis (DFA), and } \\
\text { Correlation dimension (CD). } \\
\text { Decision tree (DT), Support vector machine (SVM) } \\
\text { and K-Nearest Neighbor are the classifiers used. }\end{array}$ & $\begin{array}{c}4 \min \\
(92.11 \%)\end{array}$ \\
\hline Fujita et al., (2016) & HRV & $\begin{array}{l}\text { - HRV signals are extracted by using the } \\
\text { Pan-Tompinks algorithm. } \\
\text { - } \quad \text { Signals are denoised using a DWT-based scheme. } \\
\text { From each interval, } 12 \text { features are estimated: } \\
\text { Fuzzy entropy (FE), Renyi entropy (REnt), Hjorth } \\
\text { parameters, Tsallis entropy (Tent), and the energy } \\
\text { obtained from the 3-level decomposition, i.e., } 8 \\
\text { energy values. }\end{array}$ & $\begin{array}{c}4 \min \\
(94.7 \%)\end{array}$ \\
\hline $\begin{array}{l}\text { Amezquita-Sanchez et } \\
\text { al., (2018) }\end{array}$ & ECG & $\begin{array}{l}\text { - min interval signals are decomposed using the } \\
\text { wavelet packet transform (WPT) to generate } \\
\text { uniform frequency bands. } \\
\text { - The homogeneity index (HI) is used as feature. } \\
\text { - The classification is carried out using the } \\
\text { Enhanced Probabilistic Neural Network (EPNN). }\end{array}$ & $\begin{array}{l}20 \mathrm{~min} \\
(95.8 \%)\end{array}$ \\
\hline This work & ECG & $\begin{array}{l}\text { EMD is used to obtain the signal components } \\
\text { (IMFs). } \\
\text { - Higuchi Fractal value and Permutation Entropy } \\
\text { are used as features. } \\
\text { - } \quad \text { MLP is the classifier }\end{array}$ & $\begin{array}{c}25 \min \\
(94 \%)\end{array}$ \\
\hline
\end{tabular}

\section{Conclusions}

The existence of an SCD event generates subtle changes in the ECG signal that can be undetected due to the contained noise in the measured signal. This feature makes SCD detection a challenging task. Because of this, the earlier the detection, the better the possibility of surviving the event. This paper proposes a new methodology that combines EMD, nonlinear indices, and neural networks to predict the occurrence of an SCD event 25 min prior to the onset, using ECG signals from the MIT/BIH-SCDH and the MIT/BIH-NSR databases [41,42]. The obtained results show that the HI and PEI values of the IMF 3 and IMF 5 can be used to detect the event, as the one-way ANOVA values show that they have the most relevance. With this information, an ANN classifier was developed, achieving a $94 \%$ accuracy. Moreover, the prediction time window of an SCD event is improved by $25 \%$ and is compared with the proposal that has the largest time window [31], giving additional time to receive medical assistance and increasing the possibilities of overcoming the event without severe physical consequences.

As future work, the proposed method will be tested using a larger database and smaller time windows in order to prove the consistency of the results; also, the assessment of the efficacy of different classifiers could lead to an increase of the prediction time can lead to further improve the possibilities of mitigating the medical effects that an SCD event can have.

Author Contributions: Conceptualization, M.T.-A., C.A.P.-R., and J.P.A.-S.; Methodology, J.P.A.-S., M.V.-R., and C.A.P.-R.; Software, O.V.-L.; Validation, J.J.D.-S.-P., J.R.R.-G., and J.P.A.-S.; Formal Analysis, M.T.-A., M.V.-R., and C.A.P.-R.; Investigation, O.V.-L., J.J.D.-S.-P., and J.R.R.-G.; Writing-Original Draft Preparation, O.V.-L., M.T.-A., J.P.A.-S., and C.A.P.-R.; Writing-Review and Editing, J.J.D.-S.-P., J.R.R.-G., and M.V.-R. All authors have read and agreed to the published version of the manuscript.

Funding: This research received no external funding. 
Conflicts of Interest: The authors declare no conflict of interest.

\section{References}

1. Hernandez-Contreras, D.; Peregrina-Barreto, H.; Rangel-Magdaleno, J.; Gonzalez-Bernal, J.A.; Altamirano-Robles, L. A quantitative index for classification of plantar thermal changes in the diabetic foot. Inf. Phys. Technol. 2017, 81, 242-249. [CrossRef]

2. Yang, W.; Dall, T.M.; Tan, E.; Byrne, E.; Iacobucci, W.; Chakrabarti, R.; Loh, F.E. Diabetes diagnosis and management among insured adults across metropolitan areas in the US. Prev. Med. Rep. 2018, 10, $227-233$. [CrossRef] [PubMed]

3. Acharya, U.R.; Bhat, S.; Koh, J.E.; Bhandary, S.V.; Adeli, H. A novel algorithm to detect glaucoma risk using texture and local configuration pattern features extracted from fundus images. Compt. Biol. Med. 2017, 88, 72-83. [CrossRef] [PubMed]

4. Raghavendra, U.; Bhandary, S.V.; Gudigar, A.; Acharya, U.R. Novel expert system for glaucoma identification using non-parametric spatial envelope energy spectrum with fundus images. Biocybern. Biomed. Eng. 2018, 38, 170-180. [CrossRef]

5. Garduño-Ramón, M.A.; Vega-Mancilla, S.G.; Morales-Henández, L.A.; Osornio-Rios, R.A. Supportive noninvasive tool for the diagnosis of breast cancer using a thermographic camera as sensor. Sensors 2017, 17, 497. [CrossRef] [PubMed]

6. Gogoi, U.R.; Bhowmik, M.K.; Bhattacharjee, D.; Ghosh, A.K. Singular value based characterization and analysis of thermal patches for early breast abnormality detection. Australas. Phys. Eng. Sci. Med. 2018, 41, 1-19. [CrossRef] [PubMed]

7. Valderas, M.T.; Bolea, J.; Laguna, P.; Bailón, R.; Vallverdú, M. Mutual information between heart rate variability and respiration for emotion characterization. Phys. Meas. 2019, 40, 084001. [CrossRef] [PubMed]

8. delEtoile, J.; Adeli, H. Graph Theory and Brain Connectivity in Alzheimer's Disease. Neuroscience 2017, 23, 616-626. [CrossRef]

9. Khedher, L.; Illán, I.A.; Górriz, J.M.; Ramírez, J.; Brahim, A.; Meyer-Baese, A. Independent Component Analysis-Support Vector Machine-Based Computer-Aided Diagnosis System for Alzheimer's with Visual Support. Int. J. Neural Syst. 2017, 27, 1650050. [CrossRef]

10. Lopez-de-Ipina, K.; Martinez-de-Lizarduy, U.; Calvo, P.M.; Mekyska, J.; Beitia, B.; Barroso, N.; Ecay-Torres, M. Advances on automatic speech analysis for early detection of Alzheimer disease: A non-linear multi-task approach. Curr. Alzheimer Res. 2018, 15, 139-148. [CrossRef]

11. Duraisamy, B.; Shanmugam, J.V.; Annamalai, J. Alzheimer disease detection from structural MR images using FCM based weighted probabilistic neural network. Brain Imaging Behav. 2018, 13, 1-24. [CrossRef]

12. Amezquita-Sanchez, J.P.; Adeli, A.; Adeli, H. A new methodology for automated diagnosis of mild cognitive impairment (MCI) using magnetoencephalography (MEG). Behav. Brain Res. 2016, 305, 174-180. [CrossRef] [PubMed]

13. López-Sanz, D.; Garcés, P.; Álvarez, B.; Delgado-Losada, M.L.; López-Higes, R.; Maestú, F. Network Disruption in the Preclinical Stages of Alzheimer's Disease: From Subjective Cognitive Decline to Mild Cognitive Impairment. Int. J. Neural Syst. 2017, 27, 1750041. [CrossRef]

14. Mammone, N.; Bonanno, L.; Salvo, S.D.; Marino, S.; Bramanti, P.; Bramanti, A.; Morabito, F.C. Permutation disalignment index as an indirect, EEG-based, measure of brain connectivity in MCI and AD patients. Int. J. Neural Syst. 2017, 27, 1750020. [CrossRef] [PubMed]

15. Amezquita-Sanchez, J.P.; Mammone, N.; Morabito, F.C.; Marino, S.; Adeli, H. A novel methodology for automated differential diagnosis of mild cognitive impairment and the Alzheimer's disease using EEG signals. J. Neurosci. Meth. 2019, 322, 88-95. [CrossRef]

16. Oh, J.; Cho, D.; Park, J.; Na, S.H.; Kim, J.; Heo, J.; Shin, C.S.; Kim, J.-J.; Park, J.Y.; Lee, B. Prediction and early detection of delirium in the intensive care unit by using heart rate variability and machine learning. Phys. Meas. 2018, 39, 035004. [CrossRef] [PubMed]

17. Gao, Z.K.; Cai, Q.; Yang, Y.X.; Dong, N.; Zhang, S.S. Visibility graph from adaptive optimal kernel time-frequency representation for classification of epileptiform EEG. Int. J. Neural Syst. 2017, 27, 1750005. [CrossRef] [PubMed] 
18. Symonds, J.D.; Zuberi, S.M.; Johnson, M.R. Advances in epilepsy gene discovery and implications for epilepsy diagnosis and treatment. Cur. Op. Neurol. 2017, 30, 193-199. [CrossRef] [PubMed]

19. Acharya, U.R.; Oh, S.L.; Hagiwara, Y.; Tan, J.H.; Adeli, H. Deep convolutional neural network for the automated detection and diagnosis of seizure using EEG signals. Comp. Biol. Med. 2018, 100, 270-278. [CrossRef]

20. Atri, R.; Mohebbi, M. Obstructive sleep apnea detection using spectrum and bispectrum analysis of single-lead ECG signal. Phys. Meas. 2015, 36, 1963. [CrossRef]

21. Jonmohamadi, Y.; Poudel, G.R.; Innes, C.C.; Jones, R.D. Microsleeps are Associated with Stage-2 Sleep Spindles from Hippocampal-Temporal Network. Int. J. Neural Syst. 2016, 26, 1650015. [CrossRef] [PubMed]

22. Bruder, J.C.; Dümpelmann, M.; Piza, D.L.; Mader, M.; Schulze-Bonhage, A.; Jacobs-Le Van, J. Physiological Ripples Associated with Sleep Spindles Differ in Waveform Morphology from Epileptic Ripples. Int. J. Neural Syst. 2017, 27, 1750011, (15 pages). [CrossRef] [PubMed]

23. Karan, B.; Sahu, S.S.; Mahto, K. Parkinson disease prediction using intrinsic mode function based features from speech signal. Biocybern. Biomed. Eng. 2019, in press. [CrossRef]

24. Nilashi, M.; Ibrahim, O.; Ahmadi, H.; Shahmoradi, L.; Farahmand, M. A hybrid intelligent system for the prediction of Parkinson's Disease progression using machine learning techniques. Biocybern. Biomed. Eng. 2018, 38, 1-15. [CrossRef]

25. Ahmadlou, M.; Adeli, H. Complexity of weighted graph: A new technique to investigate structural complexity of brain activities with applications to aging and autism. Neurosci. Lett. 2017, 650, 103-108. [CrossRef] [PubMed]

26. Akar, S.A.; Kara, S.; Latifoğlu, F.; Bilgiç, V. Analysis of the complexity measures in the EEG of schizophrenia patients. Int. J. Neural Syst. 2016, 26, 1650008. [CrossRef]

27. Schmidt, M.; Schumann, A.; Müller, J.; Bär, K.-J.; Rose, G. ECG derived respiration: Comparison of time-domain approaches and application to altered breathing patterns of patients with schizophrenia. Phys. Meas. 2017, 38, 601. [CrossRef]

28. Mammone, N.; Ieracitano, C.; Adeli, H.; Bramanti, A.; Morabito, F.C. Permutation Jaccard Distance-Based Hierarchical Clustering to Estimate EEG Network Density Modifications in MCI Subjects. IEEE Trans. Neural Netw. Learn. Syst. 2018, 29, 5122-5135. [CrossRef]

29. Shafique, A.; Sayeed, M.; Tsakalis, K. Nonlinear Dynamical Systems with Chaos and Big Data: A Case Study of Epileptic Seizure Prediction and Control. In Guide to Big Data Applications. Studies in Big Data; Srinivasan, S., Ed.; Springer: Cham, Switzerland, 2018; Volume 26.

30. Ebrahimzadeh, E.; Manuchehri, M.S.; Amoozegar, S.; Araabi, B.N.; Soltanian-Zadeh, H. A time local subset feature selection for prediction of sudden cardiac death from ECG signal. Med Biol. Eng. Comp. 2018, 56, 1253-1270. [CrossRef]

31. Amezquita-Sanchez, J.P.; Valtierra-Rodriguez, M.; Adeli, H.; Perez-Ramirez, C.A. A Novel Wavelet Transform-Homogeneity Model for Sudden Cardiac Death Prediction Using ECG Signals. J. Med. Sys. 2018, 42, 176. [CrossRef]

32. Myerburg, R.J.; Junttila, M.J. Sudden cardiac death caused by coronary heart disease. Circulation 2012, 125, 1043-1052. [CrossRef] [PubMed]

33. Finocchiaro, G.; Papadakis, M.; Sharma, S.; Sheppard, M. Sudden Cardiac Death. Eur. Heart J. 2017, 38, 1280-1282. [CrossRef]

34. Pagidipati, N.J.; Gaziano, T.A. Estimating deaths from cardiovascular disease: A review of global methodologies of mortality measurement. Circulation 2013, 127, 749-756. [CrossRef] [PubMed]

35. Myerburg, R.J. Cardiac arrest and sudden cardiac death. In Heart Disease, a Textbook of Cardiovascular Medicine; W.B. Saunders: Philadelphia, PA, USA, 1992; pp. 756-789.

36. Murukesan, L.; Murugappan, M.; Iqbal, M.; Saravanan, K. Machine learning approach for sudden cardiac arrest prediction based on optimal heart rate variability features. J. Med. Imaging Health Inf. 2014, 4, 521-532. [CrossRef]

37. Murugappan, M.; Murukesan, L.; Omar, I.; Khatun, S.; Murugappan, S. Time Domain Features Based Sudden Cardiac Arrest Prediction Using Machine Learning Algorithms. J. Med. Imaging Health Inf. 2015, 5, 1267-1271. [CrossRef] 
38. Acharya, U.R.; Fujita, H.; Sudarshan, V.K.; Sree, V.S.; Eugene, L.W.J.; Ghista, D.N.; San Tan, R. An integrated index for detection of sudden cardiac death using discrete wavelet transform and nonlinear features. Knowl. Based Sys. 2015, 83, 149-158. [CrossRef]

39. Fujita, H.; Acharya, U.R.; Sudarshan, V.K.; Ghista, D.N.; Sree, S.V.; Eugene, L.W.J.; Koh, J.E. Sudden cardiac death (SCD) prediction based on nonlinear heart rate variability features and SCD index. Appl. Soft Comp. 2016, 43, 510-519. [CrossRef]

40. Ebrahimzadeh, E.; Pooyan, M.; Bijar, A. A novel approach to predict sudden cardiac death (SCD) using nonlinear and time-frequency analyses from HRV signals. PLoS ONE 2014, 9, e81896. [CrossRef] [PubMed]

41. MIT/BIH-NSR. Database. Available online: https://www.physionet.org/physiobank/database/nsrdb/ (accessed on 1 September 2019).

42. MIT/BIH-SCDH. Available online: https://physionet.org/physiobank/database/sddb/\#clinical-information/ databased (accessed on 1 September 2019).

43. Katz, M.J. Fractals and the analysis of waveforms. Comp. Biol. Med. 1988, 18, 145-156. [CrossRef]

44. Higuchi, T. Approach to an irregular time series on the basis of the fractal theory. Phys. D 1988, 31, $277-283$. [CrossRef]

45. Wang, B.Y. Detection of structural damage using fractal dimension technique. J. Vib. Shock 2005, 24, 87-88.

46. Shannon, C.E. A mathematical theory of communication. Bell Sys. Tech. J. 1948, 27, 379-423. [CrossRef]

47. Bandt, C.; Pompe, B. Permutation entropy: A natural complexity measure for time series. Phys. Rev. Lett. 2002, 88, 174102. [CrossRef] [PubMed]

48. Acharya, U.R.; Suri, S.J.; Spaan, J.A.E.; Krishnan, S.M. (Eds.) Advances in Cardiac Signal Processing; Springer: Berlin/Heidelberg, Germany, 2007.

49. Lin, C.-H.; Du, Y.-C. Fractal QRS-complexes pattern recognition for imperative cardiac arrhythmias. Digital Signal. Process. 2010, 20, 1274-1285. [CrossRef]

50. Billeci, L.; Costi, M.; Lombardi, D.; Chiarugi, F.; Varanini, M. Automatic Detection of Atrial Fibrillation and Other Arrhythmias in ECG Recordings Acquired by a Smartphone Device. Electronics 2018, 7, 199. [CrossRef]

51. Huang, N.E.; Shen, Z.; Long, S.R.; Wu, M.C.; Shih, H.H.; Zheng, Q.; Yen, N.C.; Tung, C.C.; Liu, H.H. The empirical mode decomposition and the Hilbert spectrum for nonlinear and non-stationary time series analysis. Proc. R. Soc. Lond. A Math. Phys. Eng. Sci. 1998, 454, 903-995. [CrossRef]

52. Subasi, A. Practical Guide for Biomedical Signal. Analysis Using Machine Learning Techniques; Academic Press: Cambridge, MA, USA, 2019.

53. Ghritlahre, H.K.; Prasad, R.K. Investigation of thermal performance of unidirectional flow porous bed solar air heater using MLP, GRNN, and RBF models of ANN technique. Sci. Eng. Prog. 2018, 6, 226-235. [CrossRef]

54. Lopez-Ramirez, M.; Ledesma-Carrillo, L.; Cabal-Yepez, E.; Rodriguez-Donate, C.; Miranda-Vidales, H.; Garcia-Perez, A. EMD-Based Feature Extraction for Power Quality Disturbance Classification Using Moments. Energies 2016, 9, 565. [CrossRef]

55. Murtagh, F. Multilayer perceptrons for classification and regression. Neurocomputing 1992, 2, $183-197$. [CrossRef]

56. Kopsinis, Y.; McLaughlin, S. Development of EMD-Based Denoising Methods Inspired by Wavelet Thresholding. IEEE Trans. Signal. Process. 2009, 57, 1351-1362. [CrossRef]

57. Myerburg, R.J.; Interian, A., Jr.; Mitrani, R.M.; Kessler, K.M.; Castellanos, A. Frequency of Sudden Cardiac Death and Profiles of Risk. Am. J. Cardiol. 1997, 80, 10F-19F. [CrossRef]

58. Chugh, S.S.; Reinier, K.; Teodorescu, C.; Evanado, A.; Kehr, E.; Al Samara, M.; Mariani, R.; Gunson, K.; Jui, J. Epidemiology of Sudden Cardiac Death: Clinical and Research Implications. Progr. Cardiovasc. Dis. 2008, 51, 213-228. [CrossRef]

59. Kaltman, J.R.; Thompson, P.D.; Lantos, J.; Berul, C.I.; Botkin, J.; Cohen, J.T.; Cook, N.R.; Corrado, D.; Drezner, J.; Frick, K.D.; et al. Screening for Sudden Cardiac Death in the Young. Circulation 2011, 123, 1911-1918. [CrossRef] [PubMed]

60. Rajendra Acharya, U.; Joseph, K.P.; Kannathal, N.; Lim, C.M.; Suri, J.S. Heart rate variability: A review. Med. Biol. Eng. Compt. 2006, 44, 1031-1051. [CrossRef] [PubMed] 
61. Shen, T.W.; Shen, H.P.; Lin, C.; Ou, Y. Detection and prediction of Sudden Cardiac Death (SCD) for personal healthcare. In Proceedings of the 29th Annual International Conference of the IEEE, Buenos Aires, Argentina, 22-26 August 2007; pp. 2575-2578.

62. Ebrahimzadeh, E.; Pooyan, M. Early detection of sudden cardiac death by using classical linear techniques and time-frequency methods on electrocardiogram signals. J. Biomed. Sci. Eng. 2011, 4, 699-706. [CrossRef]

(C) 2019 by the authors. Licensee MDPI, Basel, Switzerland. This article is an open access article distributed under the terms and conditions of the Creative Commons Attribution (CC BY) license (http://creativecommons.org/licenses/by/4.0/). 